Running title: Innervated human intestinal organoids.

\title{
Pluripotent Stem Cell Derived Intestinal Organoids With An Enteric Nervous
}

System

Elise Loffet ${ }^{1}$, Lisa Brossard ${ }^{1}$, Maxime M. Mahe ${ }^{1,2,3^{*}}$.

${ }^{1}$ Université de Nantes, Inserm, TENS, The Enteric Nervous System in Gut and Brain

Diseases, IMAD, Nantes, France.

${ }^{2}$ Department of Pediatric General and Thoracic Surgery, Cincinnati Children's Hospital Medical Center, Cincinnati, Ohio, USA;

${ }^{3}$ Department of Pediatrics, University of Cincinnati, Cincinnati, Ohio;

${ }^{*}$ Corresponding author:

Maxime M. Mahe: maxime.mahe@inserm.fr

Keywords: human pluripotent stem cell; intestine, enteric nervous system; vagal neural crest cell; organoid 


\begin{abstract}
The use of human pluripotent stem cells (hPSCs) and differentiation techniques offer new ways to generate specific tissue. It is now possible to differentiate hPSC into human intestinal organoids that include an enteric nervous system. Using step-wise differentiation processes, we generate innervated intestinal organoids that form three-dimensional structures bearing an epithelium, neurons and glial cells embedded in a supporting mesenchyme. Innervated organoids further develop to a complex structure with similar organization and cellular differentiation as the developing intestine. These tools open up new fields of application in the study of the development and pathophysiology of enteric neuropathies. Herein, we describe the generation of both human intestinal organoids and vagal neural crest cells from hPSC and their combination into an innervated organoid. We also discuss technical considerations for these experiments, and highlight advantages and limitations of the system.
\end{abstract}




\section{Introduction}

The digestive tract is a complex tubular organ responsible for digestive and absorptive function. In direct contact with the environment, it forms a selective barrier allowing the passage of nutrients and preventing the passage of pathogens. The digestive tract originates from two germinal layers: the endoderm and mesoderm, while ectoderm-derived cells that give rise to enteric neurons migrate into the intestinal tube after it has formed. Endoderm derived tissue will contribute to the epithelial lining of the gut. On the other hand, the mesoderm will form sublaminar layers organized in successive tunics: the submucosal layer, the muscularis composed of longitudinal and circular smooth muscles and the serosa. This anatomical architecture ensures gastrointestinal function (Thompson et al., 2018). Although digestion and the propulsion of the food are possible because of the intestinal epithelium and the muscle, the gut motor behaviors are coordinated by an intrinsic neuronal network: the enteric nervous system (ENS).

Often called the "second brain," the ENS is an integrated and autonomous nervous system that coordinates complex behaviors of the gut by controlling peristaltic movements and changing blood flow and secretion of water, electrolytes and hormones (Heanue \& Pachnis, 2007). The developed ENS is distributed along the entire length of the digestive tract in two major plexuses (i.e. myenteric and submucosal). The myenteric neurons coordinate motor behaviours of the intestinal muscle layers i.e. gut motility. Submucosal neurons control mucosal processes via local reflexes that are triggered by local distension of the intestinal wall, distortion of the mucosa, and chemical contents in the lumen (Grundy \& Schemann, 2007). These processes result from a direct neuronal activation but also involve enteroendocrine cells which, upon stimulation by luminal nutrients or forces, release mediators such as serotonin (Alcaino et al., 2018; Neunlist \& Schemann, 2014). Developmentally, the ENS originates from the ectoderm. The ectodermal contributions to the gut are derived from migrating vagal neural crest cells (vNCC) that primarily delaminate from the neural tube, with a minor contribution from the sacral neural crest cells (Nagy \& 
Goldstein, 2017). In humans, the primary gut tube is formed by 3-4 weeks of gestation, while vNCCs migrate out of the neural tube prior to week 4 . By 7 weeks of gestation, the vNCCs have colonized the entire gastro-intestinal tract to differentiate in enteric neurons and enteric glial cells (Fu et al., 2004). From week 7, enteric neuronal cell bodies coalesce into ganglia to form the aforementioned plexuses (Wallace \& Burns, 2005). Enteric neuronal projections make connections with other ganglia or with non-neuronal tissues to control the overall function of the gut. In addition to act as a mechanical support for enteric neurons, the enteric glia participates to the maintenance of intestinal homeostasis (Neunlist et al., 2013). The gut is anatomically mature by 11 weeks of gestation but the ENS becomes functional by the end of the first trimester and continues to develop thereafter (McCann et al., 2019).

The use of human pluripotent stem cells (hSPC) and the development of methods to differentiate them into various cell types and tissue lineages have made the in vitro generation of complex human tissue possible, including the generation of human intestinal organoids (HIO). Using a stepwise differentiation process that mimics embryonic intestinal development, hPSCs can be differentiated into definitive endoderm and then into hindgut spheroids that will form three dimensional intestinal structures. After 28 days in culture, HIOs possess a polarized epithelium surrounded by supporting mesenchymal cells (Spence et al., 2011). Although HIOs resemble a functional intestine, they lack an ENS. To generate innervated HIOs, hPSC-derived vagal neural crest cells (VNCC) and hindgut spheroids can be combined in a 3D human intestinal organoid with a neuronal component (HIO+ENS). These organoids are reminiscent of a normal $\mathrm{HIO}$ with the exception they are surrounded by neurons and glial cells (Workman et al., 2017). HIO+ENS offer new opportunities in the study of the development and pathophysiology of human enteric neuropathies.

Herein, we describe a method to produce innervated HIOs from hPSCs and we outline methods to perform immunostaining assays that can be used to characterize innervated 
HIOs. We include notes on technical considerations for these experiments, and highlight key advantages and limitations of the system.

\section{Applications of intestinal organoids with an enteric nervous system}

\subsection{Medical need}

Several gastrointestinal diseases have a neuropathic origin where the ENS is impaired. Common enteric neuropathies include oesophageal achalasia, gastroparesis, enteric dysmotility, intestinal pseudo-obstruction, whereas more rare forms such as Hirschsprung's disease have a genetic basis and are associated with absence of enteric neurons. Often affecting children after birth, these gastrointestinal neuropathies can occur in adults subsequently to infection, immune mediated inflammation or neurodegeneration (Westfal \& Goldstein, 2017). However, the pathogenetic mechanisms leading to enteric neuropathies are yet to be precisely understood. With the goal of generating a comprehensive map, comparisons between innervated organoids generated from control and diseased patients will enable the identification of dysregulated genes, pathways or cell interactions, to create insights into the pathogenesis of enteric neuropathies and potential treatments.

\subsection{Research need}

Our ability to understand gastrointestinal physiology in particular with the ENS has been limited to animal models and cellular models that are yet to model with fidelity human physiology. The generation of a human innervated small intestine from hPSCs constitutes an approach that offers an understanding of human cellular functions and relationships with the environment and represent a significant advance in our ability to study human gastrointestinal development, physiology and diseases. The combination of organoid models, chemical and genetic perturbations and OMIC technologies including single-cell transcriptomics, represents an exciting avenue to address cell lineages and relationships, and gene networks. In addition, the transplantation of these organoids in 
immunocompromised animals alleviate the possibilities to understand the development of higher order tissue with enhanced physiological output (Poling et al., 2018; Workman et al., 2017). These organoid systems bring us closer to the human physiology and hold great promises to understand basic gastrointestinal physiology in health and diseases. 


\section{Protocol overview}

This protocol describes the generation of innervated human intestinal organoids (HIO+ENS) from hPSCs (Figure 1). Three main steps include vagal neural crest cells (vNCC) differentiation; human intestinal organoid (HIO) differentiation, followed by HIO-vNCCs coculture in order to generate an $\mathrm{HIO}+\mathrm{ENS}$. The resulting organoid forms a 3D structure bearing a central epithelium surrounded by mesenchymal cells with enteric neurons and glial cells embedded within the mesenchyme. The overall process takes 41 days including 13 days to differentiate vNCCs, 9 days to differentiate intestinal HIOs and 28 days of HIOvNCCs co-culture.

\section{Step by step methods}

\section{1. hPSC culture and maintenance prior to differentiation}

The maintenance of high quality hPSCs is crucial for differentiation and efficient production of both vNCCs and HIOs. Although many different hPSC cell lines can be used, each cell line behaves differently in culture i.e. cell growth. Culture conditions may be adjusted regarding maintenance, frequency of cell passaging and cell density at seeding prior differentiation.

\subsubsection{Materials and reagents}

- hPSC growing feeder-free. We routinely use the ES cell line WA01 also known as H1 (WiCell) and the iPS cell line f71.019 derived from normal human adult dermal fibroblasts (Gaignerie et al., 2018).

- Matrigel (354277, hESC-Qualified, LDEV-free, Corning) 
- mTESR-1 (85850, Stem Cell Technologies)

- DMEM/F-12, HEPES (31330-038, Life Technologies)

- DPBS 1X (CS1PBS0101, Eurobio)

- StemMACS Passaging Solution XF (130-104-688, Miltenyi Biotec)

- Tissue culture-treated 6-well plate (3516, Corning)

\subsubsection{Protocol}

hPSC culture requires the pre-coating of tissue culture plates with a suitable extracellular matrix for the attachment of cells. Thus, hESC-qualified Matrigel coated 6-well plates can be prepared prior hPSC maintenance and can be stored at $4^{\circ} \mathrm{C}$ for a maximum of 5 days.

\subsubsection{Maintenance and passaging prior differentiation}

hPSC maintenance

1. Check hPSC colonies under an inverted microscope

2. Mark differentiated cells using a non-permanent marker

Note: We check for differentiated cells two days after passaging and everyday thereafter.

Pluripotent colonies present cells with a defined nucleus, they are homogeneous and have well-defined borders. Differentiated colonies present either large cells without clear colony borders or a heterogeneous zone at the center of the colony, with occasionally threedimensional structures arising from the center.

3. Aspirate both media and marked hPSC colonies using a pipette tip hooked to a vacuum pump

4. Add $1.5 \mathrm{~mL}$ of $\mathrm{mTESR}-1$ per well

5. Return the plate to the incubator at $37^{\circ} \mathrm{C}, 5 \% \mathrm{CO}_{2}$ 
hPSC passaging

1. Split cells when confluence reaches $70-80 \%$

Note: hPSCs should be passaged at least once every 7 days.

2. Identify and mark differentiated clusters of cells under an inverted microscope

3. Aspirate both media and marked areas using a pipette tip hooked to a vacuum pump

4. Wash with $1 \mathrm{~mL}$ of DPBS per well

5. Aspirate DPBS

6. Add $1 \mathrm{~mL}$ of StemMACS ${ }^{\mathrm{TM}}$ Passaging Solution XF per well

7. Incubate the plate at room temperature for 1 to 4 minutes depending on the cell line Note: cell colonies become refractive to the light under an inverted microscope when colonies start to detached from the dish.

8. Aspirate the passaging solution when colonies' border start to lift off the dish

9. Add $1 \mathrm{~mL}$ of $\mathrm{mTESR}-1$ per well

10. Scrape the colonies using a sterile $200 \mu \mathrm{L}$ pipette tip following a grid pattern Note: Using this method, colonies will be cut in even squares.

11. Gently pipette back and forth using a $1 \mathrm{~mL}$ micropipette to detach cell clumps from the dish

12. Collect cell clumps into a $15 \mathrm{~mL}$ conical tube using a $1 \mathrm{~mL}$ micropipette

13. Distribute cell clumps into a hESC-qualified Matrigel pre-coated 6-well plates using a $1: 10$ ratio.

Note: We typically split at a ratio of 1:10 and adjust it from 1:6 to 1:18, depending on colonies density and cell growth rate.

14. Move the plate back and forth to evenly distribute the clumps in the well.

Note: avoid swirling the media as colony clumps will aggregate in the center of the well, and will not grow evenly.

15. Return the plate to the incubator at $37^{\circ} \mathrm{C}, 5 \% \mathrm{CO}_{2}$

Note: hPSCs should be kept in culture for 3 to 7 days and passaged when they reach $80 \%$ confluence. 
Note: for passaging of hPSCs to begin differentiation of vNCCs, see section 3.2. For differentiation into HIOs, see section 3.3.

\subsection{Vagal Neural Crest Cells (vNCC) differentiation}

The protocol outlined here will lead to the generation of vagal neural crest cells (vNCC) in adhesion cultures in a 6-well plate format.

\subsubsection{Materials and reagents}

All solutions should be prepared fresh using sterile cell culture grade reagents.

- mTESR-1 (85850, Stem Cell Technologies)

- Collagenase, Type I (17018029, ThermoFisher) $500 \mathrm{U} / \mathrm{mL}$ working solution in mTESR-1

- Accutase (07920, Stem Cell Technologies)

- DMEM/F-12, HEPES (31330-038, Life Technologies)

- Neurobasal Medium (21103049, ThermoFisher)

- B-27 Supplement (50X) (17504-044, ThermoFisher)

- N-2 Supplement (100X) (17502-048, ThermoFisher)

- Penicillin-Streptomycin (15140122, ThermoFisher)

- Insulin (RP-10908, Invitrogen) $2 \mathrm{mg} / \mathrm{mL}$ stock in sterile $0.005 \mathrm{~N} \mathrm{HCl}$, final concentration 5 $\mu \mathrm{g} / \mathrm{mL}$

- Human recombinant Fibroblast Growth Factor basic (FGF2; 130-093-840, Miltenyi Biotec), $100 \mu \mathrm{g} / \mathrm{ml}$ stock in sterile $\mathrm{H}_{2} \mathrm{O}$, final concentration $20 \mathrm{ng} / \mathrm{ml}$

- Human recombinant Epidermal Growth Factor (EGF; 130-097-749, Miltenyi Biotec), 500 $\mu \mathrm{g} / \mathrm{ml}$ stock in sterile PBS/0.1\% Bovine Serum Albumin, final concentration $50 \mathrm{ng} / \mathrm{ml}$ 
- ROCK specific inhibitor Y27632 (ROCKi; 1254, Tocris Bioscience), $10 \mathrm{mM}$ stock in sterile $\mathrm{H}_{2} \mathrm{O}$, final concentration $10 \mu \mathrm{M}$

- GlutaMAX (35050061, ThermoFisher)

- Retinoic Acid (R2625-50MG, Sigma-Aldrich) 20 mM stock in DMSO, final concentration 2 $\mu \mathrm{M}$

- vNCC medium: 50\% DMEM/F-12, HEPES, 50\% Neurobasal Medium supplemented with 2 mM GlutaMAX, 1X B-27 and N2 supplements, Insulin, FG2, EGF and $100 \mathrm{U} / \mathrm{mL}$ Penicillin-Streptomycin

- Fibronectin (1918-FN, R\&D), $1.5 \mu \mathrm{g} / \mathrm{mL}$ final concentration in sterile DPBS

- 35x10 mm CytoOne Dish, Non-Treated (CC7672-3340, Starlab)

\subsubsection{Protocol}

\subsubsection{Generation of PSC-derived neurospheres (Day 0 to 6)}

Day 0 (neurosphere generation) (Fig. 2A)

1. Remove differentiated cells from a well of hPSCs (see 3.2.1.1)

2. Wash with $2 \mathrm{mLs}$ of DMEM/F12, HEPES

Note: Bring DMEM/F12, HEPES to room temperature for about 15 minutes and use directly.

\section{Aspirate DMEM/F12, HEPES}

4. Add $1 \mathrm{~mL}$ of collagenase Type I prewarmed at $37^{\circ} \mathrm{C}$

5. Incubate the plate for $1 \mathrm{~h}$ at $37^{\circ} \mathrm{C}, 5 \% \mathrm{CO}_{2}$

6. Confirm detachment of the colonies' border under a microscope

7. Add $5 \mathrm{mLs}$ of DMEM/F12, HEPES

8. Gently flush the well using a $1 \mathrm{~mL}$ micropipette to detach colonies 
Note: If colonies do not fully detach, gently scrape the bottom of the plate while flushing with the $1 \mathrm{~mL}$ micropipette.

9. Collect the floating cell clumps into a $15 \mathrm{mLs}$ conical tube

10. Let the clumps settle in the conical tube

11. Aspirate the supernatant without disturbing the pellet

12. Wash with $5 \mathrm{mLs}$ of DMEM/F12, HEPES

13. Repeat steps 10 to 12

14. Add $3 \mathrm{mLs}$ of vNCC media in the $15 \mathrm{mLs}$ conical tube

15. Gently resuspend the clumps using a $1 \mathrm{~mL}$ micropipette

16. Transfer $3 \mathrm{mLs}$ of cell suspension in a sterile $35 \times 10 \mathrm{~mm}$ non-treated culture dish

17. Return the dish to the incubator at $37^{\circ} \mathrm{C}, 5 \% \mathrm{CO}_{2}$

Note: The clumps will form free-floating spheres over the next day (Figure 2B).

Days 1 to 4 (daily media change) (Fig. 2B)

1. Shake the dish to regroup the neurospheres in the center of the well

2. Collect the spheres into a $15 \mathrm{mLs}$ conical tube using a $1 \mathrm{~mL}$ micropipette

3. Let the neuropsheres settle in the conical tube

4. Aspirate the supernatant without disturbing the pellet

5. Add $1.5 \mathrm{~mL}$ of vNCC media in the $15 \mathrm{mLs}$ conical tube

6. Gently resuspend the clumps using a $1 \mathrm{~mL}$ micropipette

7. Prepare the culture dish previously used by adding $1.5 \mathrm{~mL}$ of fresh vNCC media

8. Transfer $1.5 \mathrm{~mL}$ of sphere suspension from the $15 \mathrm{mLs}$ conical tube to the dish using a 1 $\mathrm{mL}$ micropipette

Note: the total volume expected per dish is $3 \mathrm{mLs}$.

9. Return the dish to the incubator at $37^{\circ} \mathrm{C}, 5 \% \mathrm{CO}_{2}$

10. Change the vNCC media daily, repeating steps 1 to 9 until day 4 
Days 4 to 6 (induction of vagal identity)

1. Prepare $3 \mathrm{mLs}$ of $\mathrm{vNCC}$ media per dish supplemented with $2 \mu \mathrm{M}$ retinoic acid

2. Shake the dish so that the neurospheres regroup in the center

3. Collect the spheres into a $15 \mathrm{mLs}$ conical tube

4. Let the neuropsheres settle

5. Aspirate out the supernatant without disturbing the pellet

6. Add $1.5 \mathrm{~mL}$ of vNCC media supplemented with $2 \mu \mathrm{M}$ retinoic acid in the $15 \mathrm{mLs}$ conical tube

7. Prepare the culture dish previously used by adding $1.5 \mathrm{~mL}$ of fresh vNCC media supplemented with $2 \mu \mathrm{M}$ retinoic acid

8. Transfer $1.5 \mathrm{~mL}$ of neurosphere suspension from the $15 \mathrm{mLs}$ conical tube to the dish using a $1 \mathrm{~mL}$ micropipette

9. Return the dish to the incubator at $37^{\circ} \mathrm{C}, 5 \% \mathrm{CO}_{2}$

10. Change the vNCC media the next day, repeating steps 1 to 9

\subsubsection{Passaging of neurospheres on fibronectin-coated plates (Day 6 to 13)}

vNCC culture requires the pre-coating of tissue culture plates with fibronectin. Thus, fibronectin coated 6-well plates must be prepared at least an hour prior to passaging using $1.5 \mu \mathrm{g} / \mathrm{mL}$ fibronectin in sterile DPBS. We recommend making 4 wells of a 6 -well plate for one $35 \times 10 \mathrm{~mm}$ dish of neurospheres. The pre-coated plates can be stored at $4^{\circ} \mathrm{C}$ for a maximum of 3 days.

Day 6 to 10

1. Shake plate to regroup the neurospheres in the center of the well

2. Pick and count spheres under a stereoscope 
Note: a collection of 25 to 50 neurospheres is recommended per fibronectin-coated well.

3. Transfer $25-50$ neurospheres into a $15 \mathrm{mLs}$ conical tube

4. Wait for the spheres to settle in the tube

5. Aspirate the supernatant without disturbing the pellet

6. Gently resuspend $25-50$ neurospheres in $1.5 \mathrm{~mL}$ of vNCC media

7. Transfer the neurospheres onto the fibronectin pre-coated plate using a final volume of 1.5 $\mathrm{mL}$ of $\mathrm{vNCC}$ media per well.

8. Shake the plate back and forth to evenly distribute the neurospheres in the well

9. Return the plate to the incubator at $37^{\circ} \mathrm{C}, 5 \% \mathrm{CO}_{2}$

Note: Neurospheres will adhere to the fibronectin-coated plate and form rosettes (Fig. 2C).

10. Change the media everyday using $1.5 \mathrm{~mL}$ vNCC media per well until day 9

Days 10 to 13 (passaging of neural crest cells)

1. Aspirate vNCC media.

2. Wash with $1 \mathrm{~mL}$ of DMEM/F12, HEPES

3. Add $1 \mathrm{~mL}$ of Accutase

4. Incubate $2 \min$ at $37^{\circ} \mathrm{C}$

Note: Confirm cell detachment under an inverted microscope. Only migrating vagal neural crest cells are picked up, the rosettes remain attached on the plate. We recommend a 2 minutes incubation with Accutase. Longer treatment will result in a detachment of the rosettes.

5. Add $5 \mathrm{mLs}$ of DMEM/F12

6. Transfer the cell suspension into a $15 \mathrm{msL}$ conical tube

7. Centrifuge at $300 \times g$ for $5 \mathrm{~min}$

8. Aspirate the supernatant without disturbing the pellet

9. Resuspend the cell pellet in $1 \mathrm{~mL}$ of vNCC media 
10. Count cells using a Malassez counting chamber or other cell counting method.

11. Seed 180000 single cells per well of a sterile tissue culture-treated 6-well plate (20 $000 \mathrm{cell} / \mathrm{cm}^{2}$ ) in $1.5 \mathrm{~mL}$ of vNCC media.

12. Shake the plate back and forth evenly to distribute the cell suspension in the well

13. Return the plate to the incubator at $37^{\circ} \mathrm{C}, 5 \% \mathrm{CO}_{2}$

Note: Cells will adhere overnight to the bottom of the 6-well plate.

14. Change the vNCC media everyday with $1.5 \mathrm{~mL}$ per well until day 13

Beyond day 13 (passaging of neural crest cells)

vNCCs can be passaged every 3-4 days. Thus, we repeat passaging of the neural crest cells from step 1 to 13 . vNCCs maintain their phenotype up to 3 passages before combination with intestinal spheroids (Fig. 2D). Although we do not typically use it, the vNCCs from the first or second passages can be frozen and thawed out using standard techniques.

\subsection{HIO differentiation and culture}

The protocol outlined here will lead to the generation of human hindgut spheroids emerging from adhesion cultures in a 24-well plate format. The spheroids will be used in combination with the vNCCs produced. We dissociate hPSC colonies into single cells rather than cell clumps to obtain a robust and reproducible spheroid generation.

\subsubsection{Materials and reagents}

All solutions should be prepared fresh using sterile cell culture grade reagents.

- mTESR-1 (85850, Stem Cell Technologies)

- Matrigel (354277, hESC-Qualified, LDEV-free, Corning) 
- ROCK inhibitor Y27632 (ROCKi; 1254, Tocris Bioscience), 10 mM stock in sterile $\mathrm{H}_{2} \mathrm{O}$, final concentration $10 \mu \mathrm{M}$

- DMEM/F-12, HEPES (31330-038, Life Technologies)

- Accutase (07920, Stem Cell Technologies)

- RPMI Media 1640 (21875-034, ThermoFisher)

- MEM Non-Essential Amino Acids Solution (100X) (NEAA, 11140-035, ThermoFisher)

- Fetal Bovine Serum (dFBS, 26140087, Life Technologies)

- Activin A (338-AC, R\&D System), $500 \mu \mathrm{g} / \mathrm{mL}$ stock in $4 \mathrm{mM} \mathrm{HCl}$, final concentration 100 $\mathrm{ng} / \mathrm{ml}$

- Human recombinant Fibroblast Growth Factor 4 (FGF4; 235-F4-01M, R\&D System), 100 $\mu \mathrm{g} / \mathrm{mL}$ stock in sterile PBS, $0.1 \%$ BSA, final concentration $500 \mathrm{ng} / \mathrm{ml}$

- Chiron (CHIR99021, 4423/10, Tocris Bioscience), reconstituted in DMSO at 10 mM stock in sterile DMSO, final concentration $3 \mu \mathrm{M}$

- Hindgut differentiation medium: RPMI Media 1640 supplemented with 1X NEAA, 2\% dFBS, $500 \mathrm{ng} / \mathrm{ml} \mathrm{FGF4} \mathrm{and} 3 \mu \mathrm{M}$ Chiron

- Nunclon Delta 24-well plates (142475, ThermoFisher)

\subsubsection{HIO culture protocol (Day 0 to 9 )}

$\mathrm{HIO}$ differentiation requires the pre-coating of tissue culture plates with Matrigel. hESCqualified Matrigel coated 24-well plates can be prepared prior differentiation and can be stored at $4^{\circ} \mathrm{C}$ for a maximum of 5 days. To seed a 24-well plate with hPSCs, we recommend using 2 wells of a 6 -well plate of hPSC at $80 \%$ confluence.

Day 0 to 2 (hPSC passaging and plating) (Fig. 3A)

1. Remove differentiated cells from a well of hPSCs (see 3.2.1.1) 
2. Wash each well to be passaged with $1 \mathrm{~mL}$ of DMEM/F12, HEPES

3. Add $1 \mathrm{~mL}$ of room temperature Accutase to each well

4. Incubate 5 minutes at $37^{\circ} \mathrm{C}$

Note: Cell clumps will detach from the bottom of the plate. The incubation time can be extended up to 7 total minutes if colonies remain attached.

5. Add $2 \mathrm{mLs}$ of DMEM/F12, HEPES

6. Pipette back and forth to dissociate and break cell clumps into single cells

7. Collect the cell suspension from all wells of a 6 -well plate in a $15 \mathrm{mLs}$ conical tube.

Note: Proceed rapidly to the following steps to avoid excessive cell death as the enzyme

Accutase is just diluted and not inactivated

8. Centrifuge for $1 \mathrm{~min}$ at $300 \times \mathrm{g}$

9. Aspirate the supernatant

10. Re-suspend the cell pellet with $1 \mathrm{~mL}$ of $\mathrm{mTESR}-1$ with ROCKi

11. Count cells using a Mallasez chamber and calculate the volume needed of cell suspension to seed 24 wells of a 24 well plate

Note: We typically seed 100000 cells per well of a 24 well plate. Therefore, we need 2,4.106 cells per 12 mLs of mTSER-1 with ROCKi.

12. Add the calculated volume of cell suspension in a $15 \mathrm{mLs}$ conical tube containing 12 mLs of mTESR-1 with ROCKi

13. Distribute $500 \mu \mathrm{L}$ of cell suspension per well of a 24 well plate, using a $1 \mathrm{~mL}$ micropipette

Note: Pipette back and forth the cell suspension in the $15 \mathrm{mLs}$ conical tube every 6 wells to avoid cell sedimentation during the cell seeding

14. Shake the plate back and forth to distribute the cells evenly in the wells

15. Return the plate to the incubator at $37^{\circ} \mathrm{C}, 5 \% \mathrm{CO}_{2}$ for 24 hours

16. Aspirate the media

17. Add $500 \mu \mathrm{L}$ of $\mathrm{mTSER}-1$ per well

18. Return the plate to the incubator at $37^{\circ} \mathrm{C}, 5 \% \mathrm{CO}_{2}$ for 24 hours (Fig. 3B) 
Day 2 to 5 (Definitive endoderm differentiation)

1. Aspirate media on day 2

2. Add $500 \mu \mathrm{L}$ of RPMI Media 1640 supplemented with $1 X$ NEAA and $100 \mathrm{ng} / \mathrm{ml}$ Activin A per well (Fig. 3C)

3. Aspirate media on day 3

4. Add $500 \mu \mathrm{L}$ of RPMI Media 1640 supplemented with 1 X NEAA, $0,2 \%$ dFBS and $100 \mathrm{ng} / \mathrm{ml}$ Activin A per well

5. Aspirate media on day 4

6. Add $500 \mu \mathrm{L}$ of RPMI Media 1640 supplemented with 1 X NEAA, $2 \%$ dFBS and $100 \mathrm{ng} / \mathrm{ml}$ Activin A per well

Day 5 to 9 (Intestinal tissue differentiation) (Fig. 3D)

1. Aspirate media

2. Add $500 \mu \mathrm{L}$ of Hindgut differentiation medium

3. Repeat steps 1 and 2 on 4 consecutive days

Note: Spheroids may appear on day 7 or 8 . In this case, use a $1 \mathrm{~mL}$ micropipette to discard the media instead of using a vacuum aspiration. When spheroids begin to emerge from the culture, continue to section 3.4 to combine spheroids with vNCCs.

\subsection{Generation of HIO+ENS}

When spheroids emerge by day 9 , vNCCs can be combined prior their inclusion in a extracellular matrix gel (Matrigel). 


\subsubsection{Materials and reagents}

- Matrigel Growth Factor Reduced (GFR) Basement Membrane Matrix, (GFR, LDEV-free , 354230, Corning)

- DMEM/F12, HEPES (31330-038, Life Technologies)

- Accutase (07920, StemCell Technologies)

- Advanced DMEM/F-12 (12634-010, ThermoFisher)

- B-27 Supplement (50X) (17504-044, ThermoFisher)

- N-2 Supplement (100X) (17502-048, ThermoFisher)

- HEPES (15630-056, ThermoFisher)

- GlutaMAX (35050-038, ThermoFisher)

- Penicillin-Streptomycin (15140122, ThermoFisher)

- Human recombinant Epidermal Growth Factor (EGF; 130-097-749, Miltenyi Biotec), 500 ug/ml stock in sterile PBS/0.1\% Bovine Serum Albumin, final concentration $50 \mathrm{ng} / \mathrm{ml}$

- Minigut media: Advanced DMEM/F-12 supplemented with 1X B27 and 1X N2 supplements, 10 mM HEPES, 2 mM GlutaMAX, $100 \mathrm{U} / \mathrm{mL}$ Penicillin-Streptomycin and 50 $\mathrm{ng} / \mathrm{ml}$ EGF

- Nunclon Delta 24-well plates (142475, ThermoFisher)

\subsubsection{Protocol}

Recovery of intestinal spheroids

1. Recover floating spheroids using a $1 \mathrm{~mL}$ micropipette under a stereo-microscope Note: While collecting spheroids, we recommend that a rough estimate of the number of spheroids is obtained, in order to determine the number of vNCCs that will be required. 
2. Collect the spheroids in a $15 \mathrm{mLs}$ conical tube. Multiple wells from a 24 well plate can be combined into one tube.

3. Wait for the spheroids to settle in the tube

4. Aspirate out media

5. Add $1 \mathrm{~mL}$ of DMEM/F12

6. Place the $15 \mathrm{mLs}$ conical tube in an incubator and proceed to the recovery of vNCCs

Recovery of vNCCs

A well of a 6-well plate of vNCCs usually contains around 0,4-0,5.106 cells. We recommend using a ratio of 1000 vNCCs per spheroid.

1. Aspirate media

2. Wash with $1 \mathrm{~mL}$ DMEM/F12

3. Add $1 \mathrm{~mL}$ Accutase

4. Incubate $2-3 \mathrm{~min}$ at $37^{\circ} \mathrm{C}$.

5. Add $5 \mathrm{mLs}$ of DMEM/F12

6. Transfer the cell suspension in a $15 \mathrm{mLs}$ conical tube.

7. Centrifuge at $300 \times g$ for $5 \mathrm{~min}$.

8. Aspirate supernatant without disturbing the pellet

9. Resuspend the cell pellet in $1 \mathrm{~mL}$ DMEM/F12

10. Count cells using a Malassez counting chamber or other cell counting method.

11. Proceed to the co-culture of intestinal spheroids and vNCCs

Co-culture of intestinal spheroids and vNCCs (Fig. 4A-D)

1. Add the required volume of vNCC suspension to the $15 \mathrm{mLs}$ conical tube containing the spheroids

Note: We recommend using a ratio of 1000 vNCCs per spheroid. 
2. Centrifuge 2 min at $300 \times g$

3. Aspirate supernatant

4. Gently resuspend the spheroids and vNCCs in ice-cold Matrigel, pipetting up and down gently to evenly mix vNCCs with spheroids.

Note: Approximately 20 to 40 spheroids per $20 \mu \mathrm{L}$ of Matrigel can be embedded. Matrigel should be kept on ice at all time to avoid polymerization.

5. Distribute $20 \mu \mathrm{L}$ droplets of ice-cold Matrigel (without vNCCs+spheroids) in the center of a 24-well plate.

Note: We realize a Matrigel bed first to avoid vNCC accumulation at the bottom of the plate.

6. Overlay the existing Matrigel drop with $20 \mu \mathrm{L}$ of hindgut spheroids+vNCCs Matrigel suspension

7. Allow Matrigel to start polymerization for 2-3 minutes at room temperature

8. Turn the cell culture plate upside-down

9. Return the plate to the incubator at $37^{\circ} \mathrm{C}, 5 \% \mathrm{CO}_{2}$ for 20 to 30 minutes

10. Overlay the Matrigel drop with $500 \mu \mathrm{L}$ of Minigut media

11. Replace media twice a week for 14 days

Passaging of innervated intestinal organoids

Organoids should be passaged after 2 weeks of culture and placed in fresh Matrigel.

1. Collect the Matrigel drop containing HIO+ENS with a $1 \mathrm{~mL}$ micropipette under a stereomicroscope using sterile culture condition

Note: $1 \mathrm{~mL}$ pipette tips can be cut to facilitate HIO+ENS removal from the Matrigel.

2. Transfer HIO+ENS in a sterile petri dish filled with DMEM/F12, HEPES

3. Under a stereomicroscope, separate $\mathrm{HIO}+\mathrm{ENS}$ from remnant Matrigel using fine forceps

4. If multiple HIO+ENS structures appear to be fused together, separate them into individual structures using fine forceps 
Note: Under the stereomicroscope, single HIO+ENS can be selected and separated by identifying a central epithelial core.

5. Collect HIO+ENS in a $15 \mathrm{mLs}$ conical tube

6. Allow HIO+ENS to settle to the bottom of the tube

Note: If HIO+ENS do not settle, briefly spin the tube at $300 \times \mathrm{g}$.

7. Aspirate supernatant

8. Resuspend $\mathrm{HIO}+\mathrm{ENS}$ in ice-cold Matrigel

Note: Approximately 5-10 HIO+ENS per $35 \mu \mathrm{L}$ of Matrigel can be embedded.

9. Distribute $35 \mu \mathrm{L}$ of Matrigel containing $\mathrm{HIO}+\mathrm{ENS}$ in the center of the well of a $24-$ well plate.

10. Allow Matrigel to start polymerization for 2-3 minutes at room temperature

11. Turn the cell culture plate upside-down

12. Return the plate to the incubator at $37^{\circ} \mathrm{C}, 5 \% \mathrm{CO}_{2}$ for 20 to 30 minutes

13. Overlay the Matrigel drop with $500 \mu \mathrm{L}$ of Minigut media

14. Replace media twice a week for 14 days

\subsection{Differentiation benchmarking}

Differentiation of cell types can be monitored using a combination of both gene or/and protein expression. For example, definitive endoderm can be assessed by controlling the proportion of CXCR4-expressing cell by flow cytometry or FOXA2 and SOX17 positive cells using immunofluorescence. Similarly, the expression of CDX2 can be verified in hindgut spheroids. Finally, the expression of CD49d, HNK-1 and P75 $5^{\text {NTR }}$ expression can be tested in vNCCs. Common markers used for benchmarking the differentiation steps for both HIOs and vNCCs are indicated in Table 1. The provided list is not exhaustive and additional markers can also be used to assess the differentiation. 


\subsection{Analysis - Fluorescent immunostaining}

The following protocol describes immunostaining on paraffin-embedded sections of intestinal organoids. This technique may be used for imaging of specific targets or for quality control during and after differentiation.

\subsubsection{Materials and reagents}

- DPBS 1X (CS1PBS0101, Eurobio)

- Paraformaldehyde, $2 \%$ diluted in DPBS

- Glycine (50046, Sigma-Aldrich)

- Histogel (HG-4000-012, ThermoFisher)

- Xylene

- Butanol

- Ethanol $100 \%, 95 \%, 90 \%, 75 \%$ and $70 \%$

- Dako target retrieval solution 10X (S1699, Agilent)

- Bovine Serum Albumin (BSA, A7030, Sigma-Aldrich)

- Triton X-100 (X100, Sigma-Aldrich)

- Tween-20 (P9416, Sigma-Aldrich)

- Horse serum (16050122, ThermoFischer)

- Rabbit anti-CDX2 antibody (ab227201, Abcam)

- Mouse Anti-E-cadherin antibody (MAB7481, R\&D Systems)

- Anti-rabbit Alexa Fluor 568 (A10042, ThermoFischer)

- Anti-rabbit Alexa Fluor 488 (A-21206, ThermoFischer)

- DAPI (D1306, ThermoFischer)

- Washing solution: DPBS supplemented with $0.5 \%$ Triton X-100, 0.05\% Tween $20,0.1 \%$ BSA 
- Blocking solution: DPBS supplemented with $0.5 \%$ Triton X-100, 0.05\% Tween $20,0.1 \%$ BSA and $10 \%$ horse serum

- Prolong glass antifade mounting solution (P36982, ThermoFisher)

- Tissue processor

- Paraffin embedding station

- Pressure cooker

\subsubsection{Protocol}

Organoid processing and paraffin section

1. Aspirate media and add $500 \mu \mathrm{L}$ of a $2 \%$ PFA solution to each well

2. Incubate for 2 hours at $4^{\circ} \mathrm{C}$

3. Aspirate PFA solution

4. Wash with $500 \mu \mathrm{L}$ of DPBS $/ 0,75 \%$ Glycine, under agitation for 10 minutes

5. Repeat the procedure 3 times

6. Retrieve HIO+ENS out of well

7. Embed 3-4 HIO+ENS in $30 \mu \mathrm{L}$ of Histogel

8. Transfer HIO+ENS into an appropriately labeled tissue processing embedding cassette.

9. Place samples in a tissue processor and proceed overnight to a standard tissue processing protocol:

$-70 \%$ Ethanol $\times 2$ for 30 min each

$-75 \%$ Ethanol for $1 \mathrm{~h}$

$-90 \%$ Ethanol for $1 \mathrm{~h}$

$-95 \%$ Ethanol for $1 \mathrm{~h}$

$-100 \%$ Ethanol $\times 2$ for $1 \mathrm{~h}$ each

$-100 \%$ Ethanol for $20 \mathrm{~min}$ 
$-100 \%$ Butanol for 20 min

-Xylene $\mathrm{x} 3$ for $1 \mathrm{~h}$ each

-Paraffin $\times 3$ for $1 \mathrm{~h}$ each

10. Remove samples from tissue processor and embed them into appropriately sized disposable base molds

11. Discard the lid of the cassette and affix the bottom portion on top of the mold creating a tissue block

12. Remove the disposable base mold from the paraffin tissue bloc

13. Section into the block slightly, exposing the desired tissue face

14. Rehydrate the tissue soaking in a bath of PBS for $1 \mathrm{~h}$ at room temperature (RT), before chilling on ice

15. Section the tissue into ribbons $3 \mu \mathrm{m}$ thick and float sections until wrinkles disappear in a tissue flotation bath

15. Mount sections on to microscope slides

16. Bake the microscope slides at $60^{\circ} \mathrm{C}$ for $1 \mathrm{~h}$

17. Store microscope slides at room temperature until further use

Staining

1. Warm microscope slides $30 \mathrm{~min}$ at $65^{\circ} \mathrm{C}$.

2. Rehydrate microscope slides by immersing them in the following series of baths:

-Xylenes x2 for 7 min each

$-100 \%$ EtOH for 5 min each

$-95 \% \mathrm{EtOH}$ for $4 \mathrm{~min}$

$-70 \% \mathrm{EtOH}$ for $3 \mathrm{~min}$

-Deionized water x2 for $2 \mathrm{~min}$

3. Perform heat induced epitope retrieval in Dako Target retrieval solution for $1 \mathrm{~min} 30 \mathrm{~s}$ at $110^{\circ} \mathrm{C}$ 
4. Permeabilize organoid sections in $0.5 \%$ Triton $X-100 / P B S$ at room temperature for $30 \mathrm{~min}$

5. Wash in wash solution for 10 min with gentle agitation

6. Repeat the procedure 2 times

7. Outline tissue sections with a hydrophobic pen to contain reagents

8. Wash slides in wash solution for 10 min each with gentle agitation

9. Repeat the procedure 3 times

10. Incubate slides with blocking solution for $1 \mathrm{~h}$ in a square bioassay dish lined with a damp paper towel to maintain humidity in the chamber

11. Aspirate blocking solution

Note: Do not wash slides.

12. Incubate slides with rabbit anti-CDX2 at [1:500] and mouse anti-CDH1 at [1:500] in blocking solution at $4{ }^{\circ} \mathrm{C}$ overnight in humidity chamber

13. Wash slides in wash solution for 10 min each with gentle agitation

14. Repeat the procedure 3 times

15. Incubate slides with donkey anti-rabbit Alexa Fluor 568 (binds to anti-CDX2 antibody) and donkey anti-mouse Alexa Fluor 488 (binds to anti-CDH1 antibody) at [1:500] in blocking solution at $4{ }^{\circ} \mathrm{C}$ overnight in a humidity chamber.

16. Wash slides in washing solution for 10 min with gentle agitation while protected from light

17. Repeat the procedure 3 times

18. Incubate slides with $1 \mu \mathrm{g} / \mathrm{mL}$ of DAPI in PBS for 10 min while protected from light

19. Wash microscope slides in PBS for $3 \times 10$ min each, while protected from light

20. Mount microscope slides with coverslips using Prolong mounting solution, or another aqueous mounting medium

Note: Wait at least 24 hours to image slides.

21. Proceed to imaging and cell counting under a fluorescent microscope (Fig. 5). 


\section{Troubleshooting \& Optimization}

Problems may occur during the differentiation and culture processes. We listed below possible solutions to overcome these issues. 


\begin{tabular}{|c|c|}
\hline Problem & Solution \\
\hline $\begin{array}{l}\text { The definitive endoderm monolayer is not } \\
\text { confluent }\end{array}$ & $\begin{array}{l}\text { You can adjust hPSC cell starting } \\
\text { density and/or control for Activin A } \\
\text { activity }\end{array}$ \\
\hline The yield of hindgut spheroid is low & $\begin{array}{l}\text { You need to adjust PSC cell starting } \\
\text { density }\end{array}$ \\
\hline Neurospheres do not adhere on the plates & $\begin{array}{l}\text { The fibronectin pre-coating is not } \\
\text { working. Plates must be coated at least } \\
\text { an hour prior the experiment }\end{array}$ \\
\hline $\begin{array}{l}\text { vNCC do not differentiate into enteric } \\
\text { neurons and glial cells within the HIO }\end{array}$ & $\begin{array}{l}\text { The number of vNCC is either too low } \\
\text { or high. Although, a range of vNCC } \\
\text { densities can be used, we recommend } \\
\text { using } 1000 \text { vNCCs per hindgut } \\
\text { spheroid }\end{array}$ \\
\hline $\begin{array}{l}\mathrm{HIO+ENS} \text { are growing flat in the culture } \\
\text { plate }\end{array}$ & $\begin{array}{l}\text { The organoids start to adhere and grow } \\
\text { on the plastic. When HIO+ENS are } \\
\text { embedded in Matrigel, we recommend } \\
\text { inverting the plate during the } \\
\text { polymerization process }\end{array}$ \\
\hline $\begin{array}{l}\mathrm{HIO}+\mathrm{ENS} \text { do not have an epithelium after } \\
28 \text { days in culture }\end{array}$ & $\begin{array}{l}\text { It is possible that mesenchymal cells } \\
\text { have outgrown the epithelium. Using } \\
\text { fine forceps, we recommend to remove } \\
\text { excess of mesenchyme during the } \\
\text { passaging procedure. }\end{array}$ \\
\hline
\end{tabular}

hPSC cell lines may present some differentiated cells if unchecked, thereby introducing unwanted cell types during the differentiation process. For these reasons, we recommend to thoroughly check and remove differentiated cells/colonies prior to starting the protocol. If the 
unwanted spontaneous differentiation increases in hPSC cultures across passages, we suggest to discard the current cells and thaw out a lower passage cryovial of undifferentiated hPSCs.

Optimal conditions for intestinal differentiation may vary for different pluripotent stem cell lines. The starting cell density is a critical aspect that needs to be tested for every hPSC line. This range is variable but a reasonable starting point is 100,000 cells per well of a 24 -well plate. We recommend testing several densities ranging from 80,000 to 140,000 cells for each differentiation. The success of differentiation can be approximated by the hindgut spheroid yield. For example, one can reasonably assume that if very few spheroids are produced, the differentiation process was sub-optimal, and the endoderm and hindgut differentiation will need to be optimized. As hPSC lines may vary across differentiation, we recommend using well-established ES lines as a gold-standard for these experiments. Thus, H1 (WA01, Wicell) or H9 (WA09, Wicell) human embryonic stem cells consistently give robust differentiation into intestinal organoids and can be used as positive controls for other iPSC lines.

Sources of reagent and lot testing are also critical for reproducibility across experiments. Recombinant protein or small molecule activity may differ. Thus, lot testing is highly recommended especially for Activin A, FGFs and CHIR99021. The testing can be performed using the aforementioned control markers during the differentiation process.

\section{Precursor and alternative techniques}

Previous group have achieved the directed differentiation of human intestinal organoids (Spence et al., 2011). Although, we primarily focused this method on the generation of small intestinal tissue from hPSCs, organoids can be differentiated to form specific regionalized areas of the gut. The directed differentiation using combined anterior-posterior signaling allows us to generate both foregut and hindgut tissues. Hence, the temporal activation of WNT/FGF/BMP/Retinoic acid/EGF signaling during the differentiation process can drive the 
tissue's fate toward oesophagus (Trisno et al., 2018), stomach (McCracken et al., 2014, 2017), jejunum- to ileum-like tissues (Tsai et al., 2017) or colon (Múnera et al., 2017). Based on the generation of human intestinal organoid, we can engineer in an ENS using enteric vNCCs. Using similar protocols, previous groups have demonstrated the directed differentiation of hPSCs into neural crest cells (NCC) (Bajpai et al., 2010; Curchoe et al., 2010) including enteric vagal neural crest cells (vNCC) (Barber et al., 2019; Fattahi et al., 2016; Lau et al., 2019). Interestingly, hPSC-derived vNCCs can be combined within different gut regions including the colon (Lau et al., 2019).

As we translate embryological development in a dish, the maturation stage of the organoids reflects the corresponding embryonic stage and is reminiscent of a fetal intestine (Finkbeiner et al., 2015). This lack of maturation may represent an issue as far as the comparability of these engineered organoids to postnatal human tissue. in vivo engraftment of $\mathrm{HIO}$ in immunocompromised mice can be used to further tissue expansion, maturation and function (Watson et al., 2014). Transplanted HIO+ENS similarly develop into a mature intestinal tissue with crypts, villi, laminated subepithelial, and mesenchymal layers. Strikingly, these $\mathrm{HIO}+\mathrm{ENS}$ exhibit similar features to the human ENS including neuronal bundles reassembling a myenteric-like plexus and a peristaltic like activity (Workman et al., 2017).

\section{Summary}

The technique described in this protocol leads to the production of innervated human intestinal organoids. The key steps include the parallel differentiation of intestinal spheroids and vNCC from hPSC, and their combination into a self-assembled innervated intestinal organoid.

Using this organoid system coupled with perturbations of developmental pathways will allow us to directly address developmental processes and disease-related mechanisms. This includes both acute and chronic enteric neuropathies. In addition, patient specific or gene edited iPSC lines, can also be used to model tissue specific diseases. For example, a 
mutation in the Paired-like homeobox 2b (PHOX2B) gene has been associated with Hirschsprung's disease and was successfully reproduced with the HIO+ENS (Workman et al., 2017).

Although $\mathrm{HIO}+\mathrm{ENS}$ are a step forward in generating intestinal tissue with greater functionality, important facets including an immune system or bacteria are absent. Strategies wherein the immune system is added to $\mathrm{HIO}+\mathrm{ENS}$ will further improve the system. The transition of a sterile gut to colonization by a microbiome, using dedicated techniques, is also an important consideration (Hill et al., 2017). Finally, surgical strategies with transplanted HIOs offer opportunities to shed light on the role of the luminal content in intestinal maturation and gut function (Mahe et al., 2017). 


\section{Acknowledgments}

This work was supported in part by ANR-17-CE14-0021 (SyNEDI to M.M.M.) and a "New Team" grant (BOGUS to M.M.M.) from the Bioregate Regenerative Medicine Cluster, University of Nantes and Région Pays de la Loire. 
Table legends:

\begin{tabular}{|c|c|c|c|}
\hline Section & Cell type & Markers & Analysis \\
\hline \multirow{4}{*}{3.2 .2 .2} & \multirow{4}{*}{ vNCCs } & HNK-1 (CD57) & qPCR, IF \\
\hline & & Neurotrophin Receptor p75 (p75NTR) & qPCR, IF \\
\hline & & SRY-Box Transcription Factor 10 (SOX10) & qPCR, IF \\
\hline & & Integrin Alpha 4 (CD49d) & FC \\
\hline \multirow{2}{*}{3.3 .2} & \multirow{2}{*}{ definitive endoderm } & SRY-Box Transcription Factor 17 (SOX17) & qPCR, IF \\
\hline & & Forkhead Box A2 (FOXA2) & qPCR, IF \\
\hline 3.3 .2 & $\mathrm{mid} / \mathrm{hindgut} \mathrm{spheroids}$ & Caudal Type Homeobox 2 (CDX2) & qPCR, IF \\
\hline \multirow{2}{*}{$3.3 / 3.4$} & \multirow{2}{*}{ epithelial cells } & Epithelial Cell Adhesion Molecule (EPCAM) & IF \\
\hline & & E-Cadherin (CDH1) & IF \\
\hline $3.3 / 3.4$ & fibroblasts & Vimentin (VIM) & IF \\
\hline $3.3 / 3.4$ & myofibroblasts & Alpha Smooth Muscle Actin (ACTA2) & IF \\
\hline \multirow{2}{*}{3.4} & \multirow{2}{*}{ glial cells } & Glial Fibrillary Acidic Protein (GFAP) & IF \\
\hline & & S100 Calcium Binding Protein B (S100B) & IF \\
\hline \multirow{2}{*}{3.4} & \multirow{2}{*}{ neurons } & Tubulin Beta 3 Class III (TUBB3) & IF \\
\hline & & Ubiquitin C-Terminal Hydrolase L1 (UCHL1) & IF \\
\hline
\end{tabular}

Table 1: Markers used for benchmarking the differentiation steps. A combination of markers can be used to confirm the efficiency and quality of differentiation using semiquantitative PCR (qPCR), immunofluorescence staining (IF) or flow cytometry (FC). 
Figure legends:

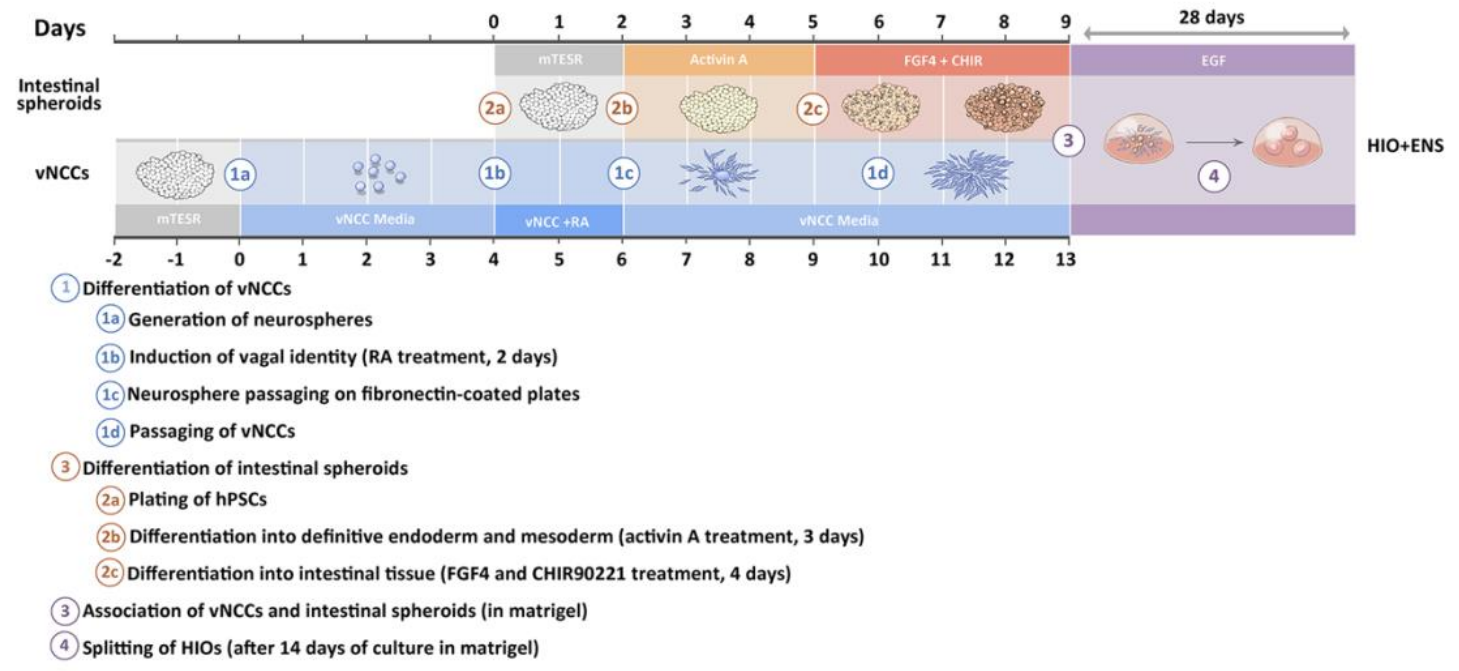

Figure 1: Innervated human intestinal organoids differentiation protocol overview. The experiment starts with the vagal neural crest cells (vNCC) differentiation, followed by the human intestinal organoid generation (HIO). Association of both vNCCs and HIOs is performed at day 13 . The combined cells result in the self-assembled intestinal organoid containing enteric glia and neurons (HIO+ENS).
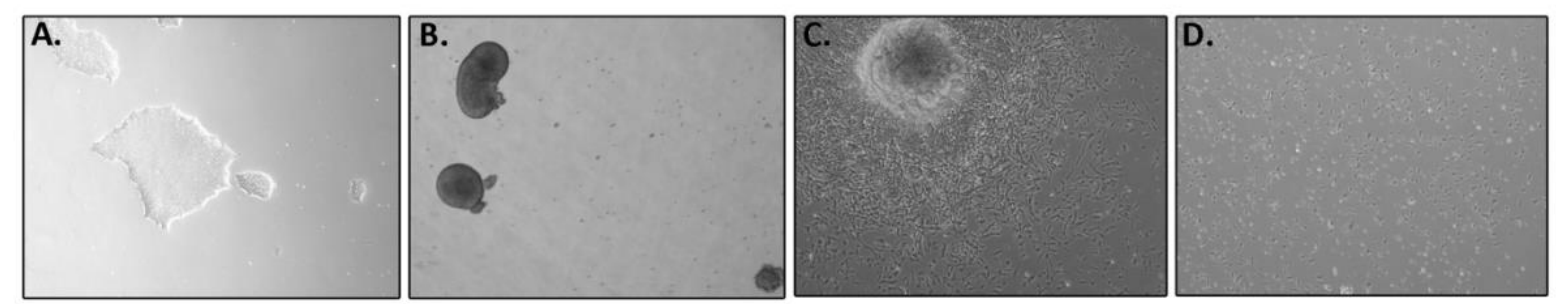

Figure 2: Representative images of vagal neural crest cell differentiation. A. hPSCs prior vNCC differentiation. B. Floating neurospheres (day 3). C. vNCCs migrating out of a neural rosette (day 9). D. vNCCs in culture after one passage (day 11). 

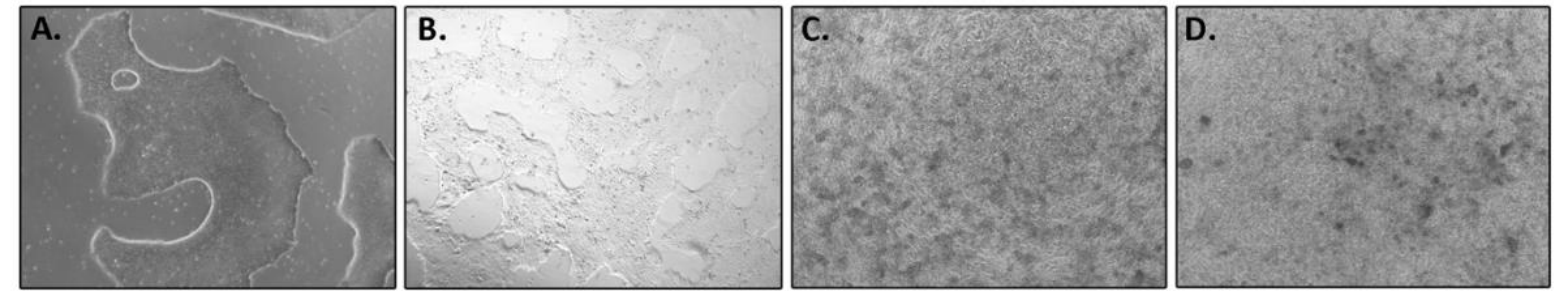

Figure 3: Representative images of hindgut spheroid differentiation.

A. hPSCs prior differentiation. B. Definitive endoderm differentiation (day 7). C. Cells forming tridimensional ridges during hindgut specification (day 10). D. End of intestinal spheroids differentiation (day 12). Spheroids are detaching from the adherent cell layer.
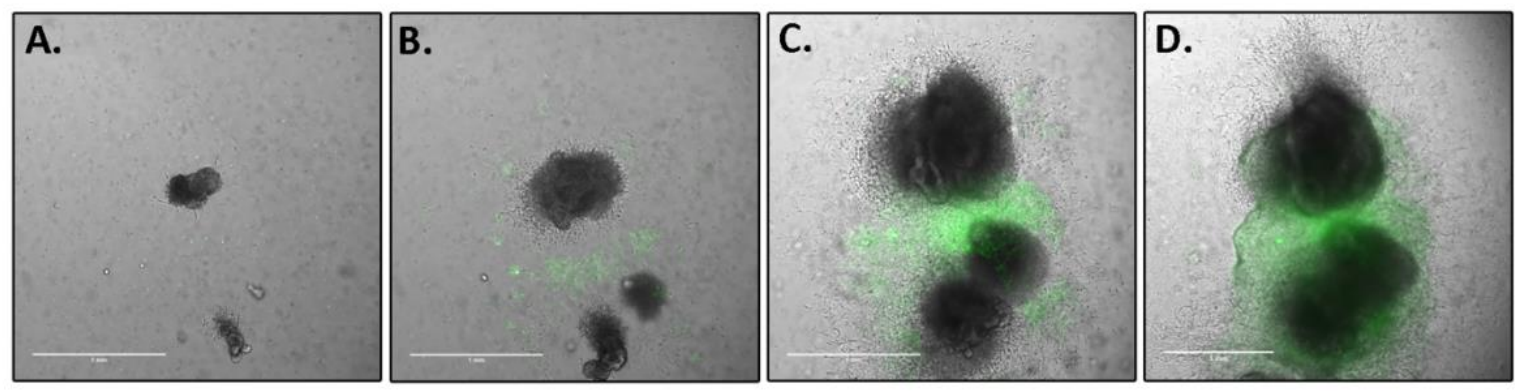

Figure 4: Growth of innervated intestinal organoid in Matrigel over 13 days.

GFP-expressing vagal neural crest cell differentiation (vNCC) are showed in green. A. Innervated intestinal organoid (HIO+ENS) a day after the combination of hindgut spheroids and vNCCs. B. HIO+ENS after 5 days in culture. C. HIO+ENS after 9 days in culture. D. $\mathrm{HIO}+\mathrm{ENS}$ after 13 days in culture prior splitting. (Scale bars: $1 \mathrm{~mm}$ ) 


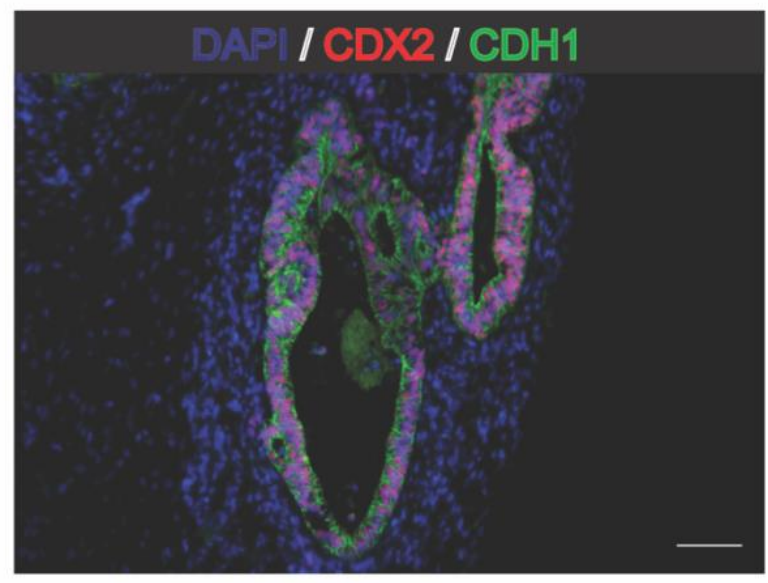

Figure 5: Immunostaining of an intestinal organoid grown for 28 days in Matrigel.

The intestinal organoid forms a central epithelium stained for E-cadherin (CDH1, green), CDX2 (red) and nuclei (DAPI, blue). A supporting mesenchyme surrounds the epithelium. (Scale bar: $50 \mu \mathrm{m})$ 


\section{References:}

Alcaino, C., Knutson, K. R., Treichel, A. J., Yildiz, G., Strege, P. R., Linden, D. R., Li, J. H., Leiter, A. B., Szurszewski, J. H., Farrugia, G., \& Beyder, A. (2018). A population of gut epithelial enterochromaffin cells is mechanosensitive and requires Piezo2 to convert force into serotonin release. Proceedings of the National Academy of Sciences of the United States of America, 115(32), E7632-E7641. https://doi.org/10.1073/pnas.1804938115

Bajpai, R., Chen, D. A., Rada-Iglesias, A., Zhang, J., Xiong, Y., Helms, J., Chang, C.-P., Zhao, Y., Swigut, T., \& Wysocka, J. (2010). CHD7 cooperates with PBAF to control multipotent neural crest formation. Nature, 463(7283), 958-962.

https://doi.org/10.1038/nature08733

Barber, K., Studer, L., \& Fattahi, F. (2019). Derivation of enteric neuron lineages from human pluripotent stem cells. Nature Protocols, 14(4), 1261-1279. https://doi.org/10.1038/s41596019-0141-y

Curchoe, C. L., Maurer, J., McKeown, S. J., Cattarossi, G., Cimadamore, F., Nilbratt, M., Snyder, E. Y., Bronner-Fraser, M., \& Terskikh, A. V. (2010). Early Acquisition of Neural Crest Competence During hESCs Neuralization. PLOS ONE, 5(11).

https://doi.org/10.1371/journal.pone.0013890

Fattahi, F., Steinbeck, J. A., Kriks, S., Tchieu, J., Zimmer, B., Kishinevsky, S., Zeltner, N., Mica, Y., El-Nachef, W., Zhao, H., de Stanchina, E., Gershon, M. D., Grikscheit, T. C., Chen, S., \& Studer, L. (2016). Deriving human ENS lineages for cell therapy and drug discovery in Hirschsprung disease. Nature, 531(7592), 105-109. https://doi.org/10.1038/nature16951

Finkbeiner, S. R., Hill, D. R., Altheim, C. H., Dedhia, P. H., Taylor, M. J., Tsai, Y.-H., Chin, A. M., Mahe, M. M., Watson, C. L., Freeman, J. J., Nattiv, R., Thomson, M., Klein, O. D., Shroyer, N. F., Helmrath, M. A., Teitelbaum, D. H., Dempsey, P. J., \& Spence, J. R. (2015). Transcriptome-wide Analysis Reveals Hallmarks of Human Intestine Development and Maturation In Vitro and In Vivo. Stem Cell Reports.

https://doi.org/10.1016/j.stemcr.2015.04.010

Fu, M., Tam, P. K. H., Sham, M. H., \& Lui, V. C. H. (2004). Embryonic development of the ganglion plexuses and the concentric layer structure of human gut: A topographical study. Anatomy and Embryology, 208(1), 33-41. https://doi.org/10.1007/s00429-003-0371-0

Gaignerie, A., Lefort, N., Rousselle, M., Forest-Choquet, V., Flippe, L., Francois-Campion, V., Girardeau, A., Caillaud, A., Chariau, C., Francheteau, Q., Derevier, A., Chaubron, F., Knöbel, S., Gaborit, N., Si-Tayeb, K., \& David, L. (2018). Urine-derived cells provide a readily accessible cell type for feeder-free mRNA reprogramming. Scientific Reports, 8(1), 14363. https://doi.org/10.1038/s41598-018-32645-2

Grundy, D., \& Schemann, M. (2007). Enteric nervous system. Current Opinion in Gastroenterology, 23(2), 121-126. https://doi.org/10.1097/MOG.0b013e3280287a23

Heanue, T. A., \& Pachnis, V. (2007). Enteric nervous system development and Hirschsprung's disease: Advances in genetic and stem cell studies. Nature Reviews. Neuroscience, 8(6), 466-479. https://doi.org/10.1038/nrn2137 
Hill, D. R., Huang, S., Nagy, M. S., Yadagiri, V. K., Fields, C., Mukherjee, D., Bons, B., Dedhia, P. H., Chin, A. M., Tsai, Y.-H., Thodla, S., Schmidt, T. M., Walk, S., Young, V. B., \& Spence, J. R. (2017). Bacterial colonization stimulates a complex physiological response in the immature human intestinal epithelium. ELife, 6. https://doi.org/10.7554/eLife.29132

Lau, S.-T., Li, Z., Pui-Ling Lai, F., Nga-Chu Lui, K., Li, P., Munera, J. O., Pan, G., Mahe, M. M., Hui, C.-C., Wells, J. M., \& Ngan, E. S.-W. (2019). Activation of Hedgehog Signaling Promotes Development of Mouse and Human Enteric Neural Crest Cells, Based on SingleCell Transcriptome Analyses. Gastroenterology, 157(6), 1556-1571.e5. https://doi.org/10.1053/j.gastro.2019.08.019

Mahe, M. M., Brown, N. E., Poling, H. M., \& Helmrath, M. A. (2017). In Vivo Model of Small Intestine. Methods in Molecular Biology (Clifton, N.J.), 1597, 229-245.

https://doi.org/10.1007/978-1-4939-6949-4_17

McCann, C. J., Alves, M. M., Brosens, E., Natarajan, D., Perin, S., Chapman, C., Hofstra, R. M., Burns, A. J., \& Thapar, N. (2019). Neuronal Development and Onset of Electrical Activity in the Human Enteric Nervous System. Gastroenterology, 156(5), 1483-1495.e6.

https://doi.org/10.1053/j.gastro.2018.12.020

McCracken, K. W., Aihara, E., Martin, B., Crawford, C. M., Broda, T., Treguier, J., Zhang, X., Shannon, J. M., Montrose, M. H., \& Wells, J. M. (2017). Wnt/ $\beta$-catenin promotes gastric fundus specification in mice and humans. Nature, 541(7636), 182-187.

https://doi.org/10.1038/nature21021

McCracken, K. W., Catá, E. M., Crawford, C. M., Sinagoga, K. L., Schumacher, M., Rockich, B. E., Tsai, Y.-H., Mayhew, C. N., Spence, J. R., Zavros, Y., \& Wells, J. M. (2014). Modelling human development and disease in pluripotent stem-cell-derived gastric organoids. Nature, 516(7531), 400-404. https://doi.org/10.1038/nature13863

Múnera, J. O., Sundaram, N., Rankin, S. A., Hill, D., Watson, C., Mahe, M., Vallance, J. E., Shroyer, N. F., Sinagoga, K. L., Zarzoso-Lacoste, A., Hudson, J. R., Howell, J. C., Chatuvedi, P., Spence, J. R., Shannon, J. M., Zorn, A. M., Helmrath, M. A., \& Wells, J. M. (2017). Differentiation of Human Pluripotent Stem Cells into Colonic Organoids via Transient Activation of BMP Signaling. Cell Stem Cell, 21(1), 51-64.e6.

https://doi.org/10.1016/j.stem.2017.05.020

Nagy, N., \& Goldstein, A. M. (2017). Enteric nervous system development: A crest cell's journey from neural tube to colon. Seminars in Cell \& Developmental Biology, 66, 94-106. https://doi.org/10.1016/j.semcdb.2017.01.006

Neunlist, M., \& Schemann, M. (2014). Nutrient-induced changes in the phenotype and function of the enteric nervous system. The Journal of Physiology, 592(Pt 14), 2959-2965. https://doi.org/10.1113/jphysiol.2014.272948

Neunlist, M., Van Landeghem, L., Mahé, M. M., Derkinderen, P., des Varannes, S. B., \& Rolli-Derkinderen, M. (2013). The digestive neuronal-glial-epithelial unit: A new actor in gut health and disease. Nature Reviews. Gastroenterology \& Hepatology, 10(2), 90-100. https://doi.org/10.1038/nrgastro.2012.221 
Poling, H. M., Wu, D., Brown, N., Baker, M., Hausfeld, T. A., Huynh, N., Chaffron, S., Dunn, J. C. Y., Hogan, S. P., Wells, J. M., Helmrath, M. A., \& Mahe, M. M. (2018). Mechanically induced development and maturation of human intestinal organoids in vivo. Nature Biomedical Engineering, 2(6), 429-442. https://doi.org/10.1038/s41551-018-0243-9

Spence, J. R., Mayhew, C. N., Rankin, S. A., Kuhar, M. F., Vallance, J. E., Tolle, K., Hoskins, E. E., Kalinichenko, V. V., Wells, S. I., Zorn, A. M., Shroyer, N. F., \& Wells, J. M. (2011). Directed differentiation of human pluripotent stem cells into intestinal tissue in vitro. Nature, 470(7332), 105-109. https://doi.org/10.1038/nature09691

Thompson, C. A., DeLaForest, A., \& Battle, M. A. (2018). Patterning the gastrointestinal epithelium to confer regional-specific functions. Developmental Biology, 435(2), 97-108. https://doi.org/10.1016/j.ydbio.2018.01.006

Trisno, S. L., Philo, K. E. D., McCracken, K. W., Catá, E. M., Ruiz-Torres, S., Rankin, S. A., Han, L., Nasr, T., Chaturvedi, P., Rothenberg, M. E., Mandegar, M. A., Wells, S. I., Zorn, A. M., \& Wells, J. M. (2018). Esophageal Organoids from Human Pluripotent Stem Cells Delineate Sox2 Functions during Esophageal Specification. Cell Stem Cell, 23(4), 501515.e7. https://doi.org/10.1016/j.stem.2018.08.008

Tsai, Y.-H., Nattiv, R., Dedhia, P. H., Nagy, M. S., Chin, A. M., Thomson, M., Klein, O. D., \& Spence, J. R. (2017). In vitro patterning of pluripotent stem cell-derived intestine recapitulates in vivo human development. Development (Cambridge, England), 144(6), 1045-1055. https://doi.org/10.1242/dev.138453

Wallace, A. S., \& Burns, A. J. (2005). Development of the enteric nervous system, smooth muscle and interstitial cells of Cajal in the human gastrointestinal tract. Cell and Tissue Research, 319(3), 367-382. https://doi.org/10.1007/s00441-004-1023-2

Watson, C. L., Mahe, M. M., Munera, J., Howell, J. C., Sundaram, N., Poling, H. M., Schweitzer, J. I., Vallance, J. E., Mayhew, C. N., Sun, Y., Grabowski, G., Finkbeiner, S. R., Spence, J. R., Shroyer, N. F., Wells, J. M., \& Helmrath, M. A. (2014). An in vivo model of human small intestine using pluripotent stem cells. Nature Medicine, 20(11), 1310-1314. https://doi.org/10.1038/nm.3737

Westfal, M. L., \& Goldstein, A. M. (2017). Pediatric enteric neuropathies: Diagnosis and current management. Current Opinion in Pediatrics, 29(3), 347-353.

https://doi.org/10.1097/MOP.0000000000000486

Workman, M. J., Mahe, M. M., Trisno, S., Poling, H. M., Watson, C. L., Sundaram, N., Chang, C.-F., Schiesser, J., Aubert, P., Stanley, E. G., Elefanty, A. G., Miyaoka, Y., Mandegar, M. A., Conklin, B. R., Neunlist, M., Brugmann, S. A., Helmrath, M. A., \& Wells, J. M. (2017). Engineered human pluripotent-stem-cell-derived intestinal tissues with a functional enteric nervous system. Nature Medicine, 23(1), 49-59.

https://doi.org/10.1038/nm.4233 